Leydi Del Rocío Silva Calpa

Promoção das propriedades óxido-redutoras da zircônia
monoclínica dopada com zinco na síntese de acetona a
partir do etanol

Tese de Doutorado

Tese apresentada como requisito parcial para obtenção do título de Doutor pelo Programa de PósGraduação em Engenharia de Materiais e de Processos Químicos e Metalúrgicos da PUC-Rio.

Orientador: Prof. Roberto Ribeiro de Avillez - PUC-Rio

Co-orientadora: Lucia Gorenstin Appel - INT 
Leydi Del Rocío Silva Calpa

\section{Promoção das propriedades óxido-redutoras da zircônia monoclínica dopada com zinco na síntese de acetona a partir do etanol}

Tese apresentada como requisito parcial para obtenção do grau de Doutor pelo Programa de PósGraduação em Engenharia de Materiais e de Processos Químicos e Metalúrgicos do Departamento de Engenharia Química e de Materiais do Centro Técnico Científico da PUC-Rio. Aprovada pela Comissão Examinadora abaixo assinada.

Prof. Roberto Ribeiro de Avillez

Orientador e Presidente Departamento de Engenharia Química e de Materiais - PUC Rio

Dra. Lucia Gorenstin Appel Co-orientadora Instituto Nacional de Tecnologia - INT

Prof. Rogério Navarro Correia de Siqueira Departamento de Engenharia Química e de Materiais - PUC Rio

Dr. Alexandre Barros Gaspar Instituto Nacional de Tecnologia - INT

Profa. Cristiane Assumpção Henriques Universidade do Estado do Rio de Janeiro - UERJ

Dra. Sonia Letichevsky Departamento de Engenharia Química e de Materiais - PUC-Rio

Prof. José Eugenio Leal Coordenador Setorial de Pós-Graduação do Centro Técnico Científico da PUC-Rio 
Todos os direitos reservados. É proibida a reprodução total ou parcial do trabalho sem autorização da universidade, da autora e do orientador.

\section{Leydi Del Rocío Silva Calpa}

Graduo-se em Química na Universidad de Nariño (Colômbia), onde foi integrante do grupo de pesquisa em Materiais Funcionais e Catálise. Mestre em Engenharia dos Materiais, Processos Químicos e Metalúrgicos pela Pontifícia Universidade Católica de Rio de Janeiro PUCRio. Possui experiência em pesquisa relacionada com materiais cerâmicos nanoestruturados e suas aplicações em catálise e remediação ambiental.

Ficha Catalográfica

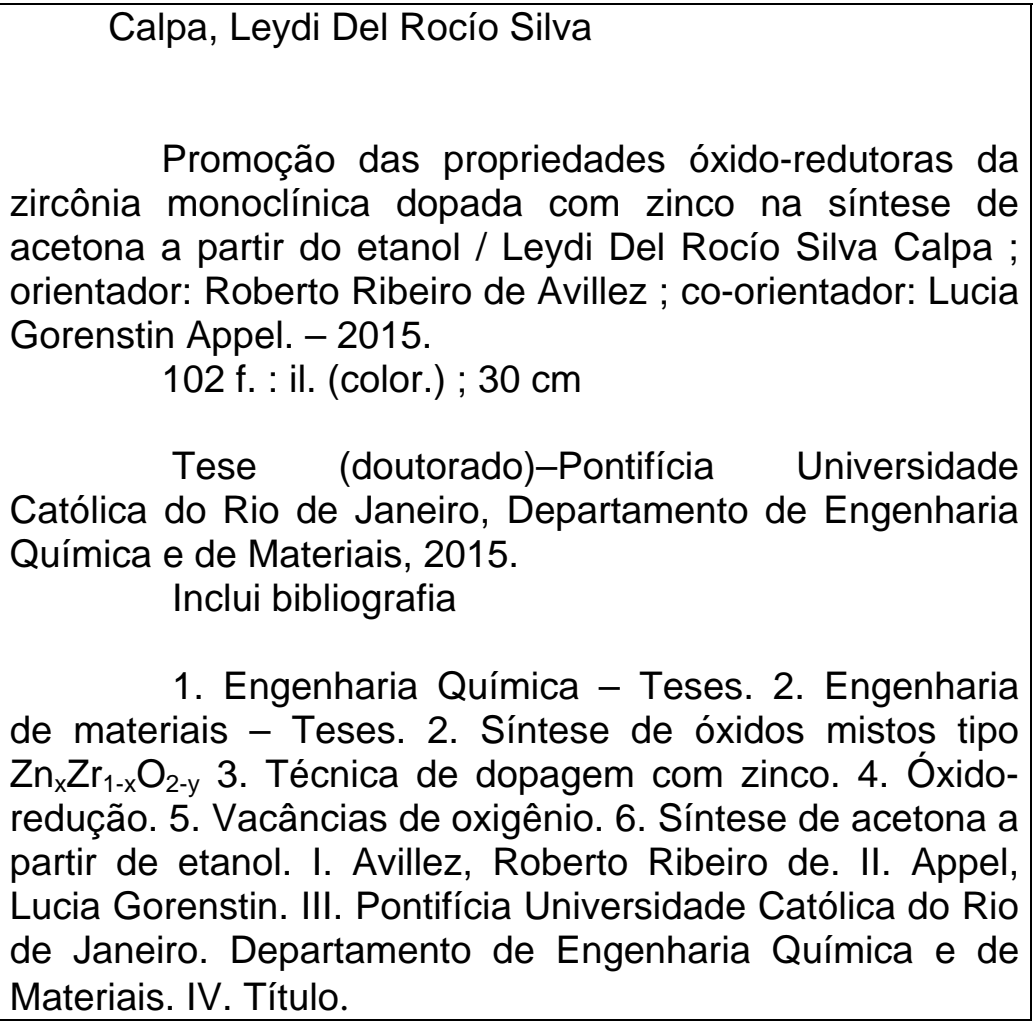

CDD: 620.11 


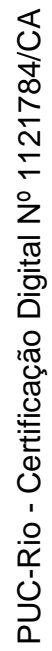

Com amor, dedico este trabalho à minha família. 


\section{Agradecimentos}

Agradeço ao meu orientador, o prof. Roberto de Avillez e à minha co-orientadora, a Dra. Lucia Gorenstin Appel pela orientação, dedicação e apoio no desenvolvimento do pressente trabalho.

Ao pessoal do laboratório CENANO e a todo o pessoal técnico do Laboratório de Catálise - LACAT do Instituto Nacional de Tecnologia, INT; Ao pessoal dos laboratórios de Física, Química e Engenharia Química e de Materiais da PUCRio; Ao Odivaldo Cambraia do laboratório de física do CBPF, e a todas as pessoas que colaboraram na realização das análises que fizeram possível o presente estudo.

À CNPq, CAPES, FAPERJ e à PUC-Rio, pelos auxílios concedidos, sem os quais este trabalho não poderia ter sido realizado.

Aos professores que fizeram parte da comissão examinadora.

Á Clarissa Rodrigues e à Priscila Zonetti, por todos seus valiosos aportes ao longo deste processo.

À Alicia Cristina, por que mesmo na distância, todos seus comentários cheios de sabedoria me encorajam e fortalecem.

Ao Cesar e à Greis, minha família no Rio, por ter ficado comigo sempre, nos bons momentos e principalmente nos mais difíceis.

Á minha família e a todos meus amigos, pelo grande amor e apoio constante. 


\section{Resumo}

Calpa, Leydi Del Rocío Silva; de Avillez, Roberto Ribeiro; Appel, Lucia Gorenstin; Promoção das propriedades óxido-redutoras da zircônia monoclínica dopada com zinco na síntese de acetona a partir do etanol. Rio de Janeiro, 2015. 102p. Tese de Doutorado - Departamento de Engenharia Química e de Materiais, Pontifícia Universidade Católica de Rio de Janeiro.

Esta tese descreve a síntese de um óxido misto de $\mathrm{Zn}$ e $\mathrm{Zr}\left(\mathrm{Zn}_{\mathrm{x}} \mathrm{Zr}_{1-\mathrm{x}} \mathrm{O}_{\mathrm{y}}\right)$, mediante uma técnica ainda não reportada e a utilização deste material na síntese de acetona a partir de etanol. O objetivo foi desenhar um catalisador com propriedades óxido-redutoras e capacidade de se autorregenerar após cada ciclo. A zircônia monoclínica $\left(m-\mathrm{ZrO}_{2}\right)$ é um óxido já usado em catálise devido às suas propriedades ácido-básicas, mas com fracas propriedades óxido-redutoras. Estas últimas poderiam ser promovidas mediante dopagem substitucional deste material. A inserção de pequeníssimas quantidades de $\mathrm{Zn}$ na rede da $m-\mathrm{ZrO}_{2}$, mostraram um incremento apreciável na sua redutibilidade. O material obtido foi caracterizado por diversas técnicas e testado na obtenção de acetona a partir de etanol. O resultado indicou que a dopagem permitiu a formação de vacâncias de oxigênio, as quais promoveram a mobilidade do oxigênio até a superfície e, com isso, aumentaram redutibilidade do material. A seletividade da reação de conversão de etanol teve como produto maioritário a acetona. Foi demostrado que a regeneração do catalisador se deve a espécies oxidantes provenientes da dissociação da água presente no médio reacional; esta dissociação ocorreu nas vacâncias superficiais. Assim, demonstrou-se que a técnica desenvolvida no presente estudo, além de simples, resultou efetiva na síntese do óxido misto de Zn e Zr, um catalisador de composição simples, capaz de conduzir os diferentes passos do mecanismo na síntese de acetona. Considera-se que esta é a primeira etapa na sua viabilização comercial.

\section{Palavras-chave}

Síntese de óxidos mistos tipo $\mathrm{Zn}_{\mathrm{x}} \mathrm{Zr}_{1-\mathrm{x}} \mathrm{O}_{\mathrm{y}}$; técnica de dopagem com zinco; óxido-redução; vacâncias de oxigênio; síntese de acetona a partir de etanol. 


\section{Abstract}

Calpa, Leydi Del Rocío Silva; de Avillez, Roberto Ribeiro (Advisor); Appel, Lucia Gorenstin (Co-advisor); Promotion of monoclinic zirconia redox properties by doping with zinc for the acetone synthesis from ethanol. Rio de Janeiro, 2015. 102p. PhD thesis - Departamento de Engenharia Química e de Materiais, Pontifícia Universidade Católica of Rio de Janeiro.

This thesis describes the synthesis process of a mixed oxide with $\mathrm{Zn}$ and $\mathrm{Zr}$ $\left(\mathrm{Zn}_{\mathrm{x}} \mathrm{Zr}_{1-\mathrm{x}} \mathrm{O}_{\mathrm{y}}\right)$, through a not yet published technique, as well as the utilization of this material in the acetone synthesis from ethanol. The mean goal was to design a catalyst with enhanced oxy-reduction properties and capability for selfregeneration after each cycle. Monoclinic zirconia $\left(m-\mathrm{ZrO}_{2}\right)$ is already used in catalysis due to its acidic-basic properties, although its oxy-reduction properties are negligible. The enhancement of the oxy-reduction properties can be reached by substitutional doping of this material with a lower oxidation state metal. The insertion of very small quantities of $\mathrm{Zn}$ in the $m-\mathrm{ZrO}_{2}$ lattice, showed a high increment of its reducibility. The mixed oxides obtained were characterized by several techniques and catalytically tested in the acetone synthesis from ethanol. The results showed that doping allows the formation of oxygen vacancies, which allow oxygen mobility and therefore, the enhancement of reducibility. The reaction selectivity had acetone as majority product. It was shown that water is also produced and that it is dissociated in the oxygen vacancies generating oxidant species. The catalysts regeneration occurs due to those oxidant species. Therefore, it was demonstrated that the technique developed in this study was easy and effective in the synthesis of the mixed oxide with $\mathrm{Zn}$ and $\mathrm{Zr}$, a catalyst with simple composition able to conduct every step of the acetone synthesis. This is considered the first step in the commercial feasibility of this material.

\section{Keywords}

Mixed oxides type $\mathrm{Zn}_{\mathrm{x}} \mathrm{Zr}_{1-\mathrm{x}} \mathrm{O}_{\mathrm{y}}$ synthesis; zinc doping technique; oxyreduction; oxygen vacancies; acetone synthesis from ethanol. 


\section{Sumário}

1 Introdução 16

2 Revisão bibliográfica $\quad 18$

$\begin{array}{lr}\text { 2.1. Produção de acetona a partir de etanol } & 18\end{array}$

2.2. Óxidos mistos e seu uso na catálise 26

2.3. Zircônia monoclínica $m-Z_{r_{2}} \quad 26$

2.3.1. Promoção das propriedades de óxido redução 27

2.3.2. Soluções sólidas substitucionais 28

2.4. Óxidos mistos de $\mathrm{Zn}$ e $m-\mathrm{ZrO}_{2}\left(\mathrm{Zn}_{\mathrm{x}} \mathrm{Zr}_{1-\mathrm{x}} \mathrm{O}_{2-\mathrm{y}}\right)$

2.5. Técnicas comumente usadas na síntese de óxidos mistos 30

2.5.1. Coprecipitação 30

2.5.2. Método de mistura física 32

2.5.3. Método de sal fundido 33

2.5.4. Rota de padrão rígido ou de espaço confinado 34

2.5.5. Método sol-gel 35

2.5.6. Método sol-gel modificado 36

2.5.7. Preparação assistida por surfactantes 37

2.5.8. Impregnação a umidade incipiente ou impregnação em seco 38

2.6. Caracterização de óxidos mistos 39

2.6.1. Análise de vacâncias por Espectroscopia de ressonância paramagnética de elétrons (EPR). 40

2.6.2. Dessorção a Temperatura Programada (TPD) e Redução a Temperatura Programada (TPR). 43

2.6.3. Reação reversa de deslocamento da água, RWGS, como reação modelo na medida da redutibilidade. 44

3 Objetivo $\quad 45$ 
4.1. Preparação do catalisador 46

4.2. Caracterização dos catalisadores 47

4.2.1. Determinação de área específica pela técnica de BET 47

4.2.2. Análise química por ICP-AES e XPS 47

4.2.3. Análise cristalográfica por difração de raios- $X \quad 48$

4.2.4. Análise química estrutural por espectroscopia Raman 48

4.2.5. Determinação da presença de vacâncias por EPR 49

4.2.6. Determinação da dissociação de moléculas de água por TPD- $\mathrm{H}_{2} \mathrm{O}$

4.2.7. Microscopia eletrônica de transmissão, MET 51

4.2.8. Medida de redutibilidade mediante TPR- $\mathrm{H}_{2}$

4.2.9. Análise das propriedades óxido-redutoras por TPD-etanol utilizando o catalisador reduzido e acompanhamento DRIFTS

4.2.10. Análise das propriedades óxido-redutoras por TPD-etanol utilizando o catalisador oxidado e acompanhamento DRIFTS e MS 53 4.2.11. Propriedades óxido-redutoras e a catálise da reação reversa de deslocamento de água (RWGS) 54

4.2.12. Determinação da basicidade superficial por TPD-CO $\mathrm{CO}_{2} \quad 55$

4.2.13. Determinação das propriedades ácidas por TPD-NH $\mathrm{NH}_{3} \quad 56$

4.3. Desempenho dos óxidos sintetizados como catalisadores na reação de síntese de acetona a partir de etanol.

5.1. Caracterização dos catalisadores 58

5.1.1. Determinação de área específica mediante a técnica BET 58

5.1.2. Análise química por ICP-AES e XPS 58

5.1.3. Análise cristalográfica por difração de raios-X 60

5.1.4. Análise química estrutural por espectroscopia Raman 63

5.1.5. Determinação da presença de vacâncias por EPR 66

5.1.6. Determinação da dissociação de moléculas de água mediante 
5.1.7. Microscopia eletrônica de transmissão, MET 69

5.1.8. Medida de redutibilidade pela técnica de TPR- $\mathrm{H}_{2}$

5.1.9. Análise das propriedades óxido-redutoras por TPD-etanol utilizando o catalisador reduzido e acompanhamento DRIFTS

5.1.10. Análise das propriedades óxido-redutoras por TPD-etanol utilizando o catalisador oxidado e acompanhamento DRIFTS e MS $\quad 74$ 5.1.11. Reação de deslocamento de água reversa, RWGS. 81

5.1.12. Determinação das propriedades ácidas e básicas 82

5.2. Desempenho dos catalisadores sintetizados na reação de geração de acetona a partir de etanol 83

5.2.1. Consideração sobre as etapas do mecanismo reacional 88

6 Conclusões 90

7 Recomendações para trabalhos futuros 92

8 Referências bibliográficas 93

9 Apêndice 99

9.1. Difractogramas indicando o respectivo ajuste Rietveld. 99

9.2. Espectros XPS indicando valores de energia de ligação do zinco101

9.3. Deconvolução dos espectros de TPD-CO ${ }_{2} .101$

9.4. Deconvolução dos espectros de TPD-NH ${ }_{3}$. 


\section{Lista de Figuras}

Figura 1 - Representação esquemática dos principais produtos

químicos derivados do etanol. Diagrama baseado em Appel $(2012)^{4}$...... 18

Figura 2 - Obtenção de acetona a partir de etanol. Rota A do

mecanismo proposto por Murthy et al. (1988). ${ }^{8}$

Figura 3 - Obtenção de acetona a partir de etanol. Rota B do mecanismo proposto por Murthy et al. (1988). ${ }^{8}$

Figura 4 - Obtenção de acetona a partir de etanol. Mecanismo

proposto por Nakajima, et al. (1994). ${ }^{3}$

Figura 5 - Obtenção de acetona a partir de etanol. Mecanismo

proposto por Yee et al. (1999), ${ }^{10}$ empregando $\mathrm{CeO}_{2}$.

Figura 6 - Obtenção de acetona a partir de etanol. Mecanismo

proposto por Yee et al. (1999), ${ }^{10}$ empregando $\mathrm{Pd} / \mathrm{CeO}_{2}$

Figura 7 - Obtenção de acetona a partir de etanol. Mecanismo proposto por Nishiguchi et al. (2005) ${ }^{7}$

Figura 8 - Representação esquemática dos níveis energéticos para um elétron livre em função de um campo magnético aplicado, B, indicando absorção EPR. Baseado em Weil \& Bolton (2007) $)^{50}$

Figura 9 - Representação esquemática de um espectro de absorção convencional, (esquerda), e de um espectro de absorção

EPR (direita). Baseado em Weil \& Bolton (2007) ${ }^{50}$

Figura 10 - Representação esquemática do pré-tratamento realizado nas amostras prévia análise por EPR, TPD- $\mathrm{H}_{2} \mathrm{O}$, TPD-etanol, TPR e RWGS

Figura 11 - Representação esquemática do pré-tratamento realizado nas amostras prévia análise por TPD-CO 2 e TPD-NH

Figura 12 - Representação esquemática da análise por EPR...................50

Figura 13 - Representação esquemática da análise por TPD- $\mathrm{H}_{2} \mathrm{O}$..........51

Figura 14 - Representação esquemática da análise por TPR- $\mathrm{H}_{2} \ldots \ldots \ldots \ldots . . .52$

Figura 15 - Representação esquemática da análise por TPD-etanol

sobre a amostra reduzida.

Figura 16 - Representação esquemática da análise por TPD-etanol 
sobre a amostra oxidada.

Figura 17 - Representação esquemática da análise das amostras na reação RWGS.

Figura 18 - Representação esquemática da análise por TPD-CO $\mathrm{CO}_{2} \ldots \ldots . . .55$

Figura 19 - Representação esquemática da análise por TPD-NH $\mathrm{NH}_{3}$... 56

Figura 20 - Representação esquemática do procedimento nos testes

catalíticos na reação de obtenção de acetona a partir de etanol.

Figura 21 - Difratograma da amostra 0,7_Zn indicando o ajuste da fase $\mathrm{m}-\mathrm{ZrO}_{2}$ e o respetivo erro experimental calculado pelo método de Rietveld, usando o programa TOPAS Academic 4.1 (Alan Coelho, $2007)^{55}$

Figura 22 - Variação do volume de célula unitária da fase monoclínica com a adição de $\mathrm{Zn}$

Figura 23 - Espectros Raman de alta resolução, indicando ausência

da fase $\mathrm{ZnO}$ nas amostras sintetizadas.

Figura 24 - Espectros EPR para as amostras m-ZrO $\mathrm{Zr}_{2}$ e 0,7_Zn nos seus estados oxidados e reduzidos, indicando sinais característicos de vacâncias após dopagem

Figura 25 - Perfis de MS do fragmento hidrogênio obtidos durante o TPD- $\mathrm{H}_{2} \mathrm{O}$ sobre as amostras $0,7 \_\mathrm{Zn}$ e $\mathrm{m}-\mathrm{ZrO}_{2}$

Figura 26 - Micrografias MET obtidas com microscópio operando em 200 kV. Esquerda) Amostra m- $\mathrm{ZrO}_{2}$ em 80.000X, direita) e 400.000X ...66 69 Figura 27 - Micrografia MET obtidas com microscópio operando em 200 kV. Amostra m-ZrO 2 em 400.000X, exibindo distâncias interplanares de 3,2 $\AA$ e planos paralelos 69

Figura 28 - Micrografia MET obtida com microscópio operando em 200 kV. Amostra 0,7_Zn, 400.000X. Direita) imagem original, esquerda) planos coloridos inseridos na mesma imagem da direita, indicando a contração interplanar nos contornos da partícula. 70

Figura 29 - Perfis de TPR das quatro amostras sintetizadas medidos entre 35 e $450^{\circ} \mathrm{C}$.

Figura 30 - Hidrogênio consumidos por massa de cada catalisador no processo de TPR de 30 a $450^{\circ} \mathrm{C}$.... 
Figura 31 - Espectros de vibração IV das espécies adsorvidas nas amostras m-ZrO 2 e 0,7_Zn na adsorção de etanol em três temperaturas diferentes.

Figura 32 - Espectros DRIFTS obtidos durante o TPD-etanol sobre as amostras a) m-ZrO $\mathrm{rO}_{2}$ e b) 0,7_Zn. Região característica de espécies etóxido.

Figura 33 - Espectros DRIFTS obtidos durante o TPD-etanol sobre as amostras a) m-ZrO $\mathrm{rO}_{2}$ e b) 0,7_Zn. Região característica de espécies acetato. 75

Figura 34 - Espectros MS obtidos durante o TPD-etanol sobre as amostras 0,7_Zn e m-ZrO

Figura 35 - Taxas de conversão de $\mathrm{CO}_{2}$ na reação de RWGS empregando as amostras estudadas.

Figura 36 - Seletividade em isoconversão (40\%) na reação de síntese de acetona a partir de etanol a $400^{\circ} \mathrm{C}$, utilizando os óxidos mistos e a $\mathrm{m}-\mathrm{ZrO}_{2}$. 84

Figura 37 - Seletividade em isoconversão (60\%) na reação de síntese de acetona a partir de etanol a $400^{\circ} \mathrm{C}$, utilizando diversos catalisadores.

Figura 38 - Seletividade da reação de conversão etanol-acetona com a mudança da temperatura, usando $50 \mathrm{mg}$ do catalisador $0,7 \_\mathrm{Zn}$. Figura 39 - Seletividade e conversão de etanol a $400^{\circ} \mathrm{C}$ em função da velocidade espacial, WHSV, utilizando o catalisador 0,7 Zn. 88 Figura 40 - Esquema reacional considerando as etapas envolvidas na produção de acetona a partir de etanol utilizando o catalisador 0,7_Zn. .89 


\section{Lista de Tabelas}

Tabela 1 Nomenclatura dos óxidos mistos; área específica; Razões atômicas Zn/Zr medidas por ICP-AES e XPS e energias de ligação (BE) determinadas por XPS 59

Tabela 2 Parâmetros cristalográficos determinados após refinamento Rietveld dos dados de DRX, usando o programa TOPAS Acadêmico 4.1. (Alan Coelho, 2007) ${ }^{55}$ 62

Tabela 3 - Números de onda $\left(\mathrm{cm}^{-1}\right)$ teóricos associados às bandas de vibração e os valores experimentais observados nos espectros Raman dos óxidos sintetizados 65

Tabela 4 - Vibrações DRIFTS das bandas de absorção de espécies etóxido e acetato calculados no presente estudo e reportados na bibliografia para diversos materiais. 76

Tabela 5 - Densidade de sítios básicos e ácidos fracos (f), médios $(\mathrm{m})$, fortes $(\mathrm{F})$ e totais $(\mathrm{T})$ 


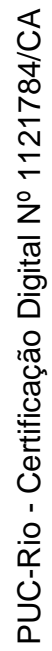

A simplicidade é o último grau de sofisticação. Leonardo da Vinci 